\title{
To Find Location of Optic Disc in Digital Fundus Images
}

\author{
Miss. Tejaswini S. Mane ${ }^{1}$, Mr. Aniket V. Kale ${ }^{2}$ \\ Department of Electronics, TKIT, Warnanagar, Shivaji University, Kolhapur ${ }^{1}$ \\ Asst. Professor, Electronic and Telecommunication, DACOE, Karad, India ${ }^{2}$
}

\begin{abstract}
Optic disc (OD) location plays an important role in automatic diagnosis of diabetic retinopathy. Medical image analysis and processing has great significance in the field of medicine, especially in non-invasive treatment and clinical study. Changes in optic disc shape and area may indicate disease processes, particularly glaucoma, and accurate identification of the disk boundary may be used to quantify changes. In this paper we are presenting the preprocessing step which will be beneficial further to calculate the cup to boundary ratio of optic disc. This paper presents technique for finding location of optic disc from digital retinal image. The optic disc pixel (ODP) should first determine for further processing. The optic disc pixel (ODP) can be further used for finding location of optic disc. Three methodologies for finding optic disc pixel and finally one voting algorithm for final optic disc pixel are presented in this paper.
\end{abstract}

Keywords: optic disc pixel (ODP), maximum variance method, maximum difference method, low pass filter method.

\section{INTRODUCTION}

Diabetic retinopathy (DR) is a chronic disease which nowadays constitutes the primary cause of blindness in people of working age in the developed world.[1].computer diagnosis is called for to allow detection of early signs of diabetic million patients with diabetes every year[8]. Retinopathy in The benefits that a system for automatically detect early signs of this disease would provide have been widely studied and assessed positively by experts [2]. It is caused by damage in blood vessels in retina. This is important for find the optic disc pixel from given retinal fundus image. The aim of this work is to finding location of optic disc innretinal image. It needs as initial information the coordinates of a pixel located within the OD. A simple but reliable and very fast OD location methodology is also proposed to obtain the required OD pixel.

OD segmentation is also relevant for automated diagnosis of other ophthalmic pathologies. One of them and maybe the most noteworthy is Glaucoma. It is the second most common Cause of blindness worldwide. Glaucoma is identified by recognizing the changes in shape, color, or depth that it Produces in the OD. Thus, its segmentation and analysis can be used to detect evidence of Glaucoma automatically.

A computer-aided fundus image analysis could provide an immediate detection and characterization of retinal features prior to specialist inspection. With the increasing size and number of medical images of eye, the use of computers in facilitating their processing and analysis has become necessary. Optic disc or optic nerve head is the point of exit for ganglion cell axons leaving the eye. The optic disc represents the beginning of optic nerve and is the point where the axons of retinal ganglion cells come together. A bio microscopic exam can give an indication of health of optic nerve.

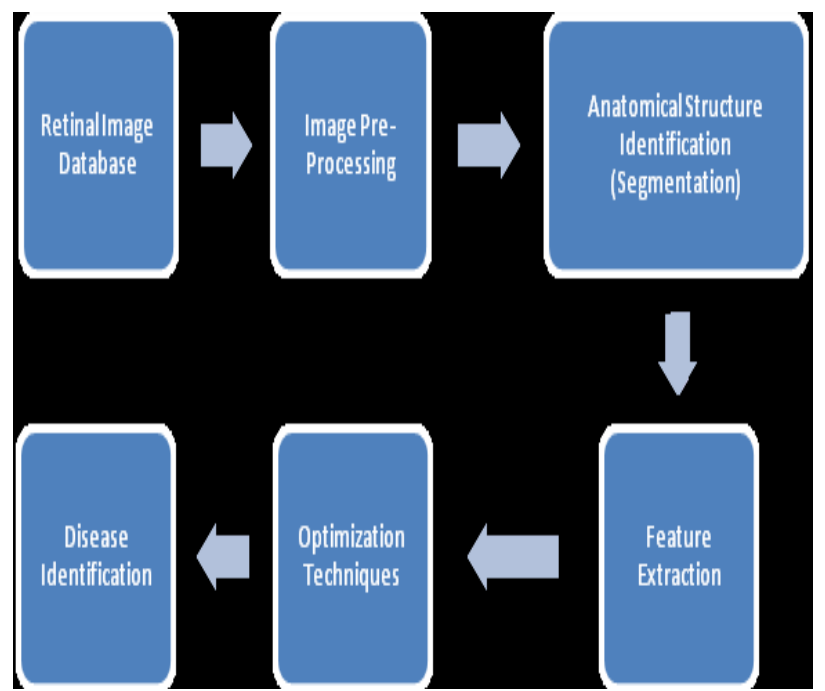

Fig.1: Automated Disease Identification System

Now days in human being the eye problems occurred in a huge amount. In which blindness comes due to optic disc. So detection optic disc is a very important research in medical science. Optic disc (OD) detection is an important step in developing systems for automated diagnosis of various serious ophthalmic pathologies.

This paper presents a new methodology for finding the ODP from digital retinal images. It requires a pixel located within the OD as initial information. For this purpose, a location methodology based on a voting-type algorithm is also proposed. From this or by using this algorithm we 
will detect the exact location of the Optic Disc.

Optic disc is a bright area on the right side of the eye where the blood vessels converges Foracchia et al. [4] It is circular area in the black of eye where the optic nerve connect to the retina called as optic disc .Generally optic disc are orange to pink in colour .Optic disc is no light sensitive spot formed in the eye that spot also called as a blind spot. In color fundus images, the OD usually appears as a bright yellowish region, although this feature may also experience significant variations.

\section{OVERVIEW OF PAPER}

First of all we have to find the ODP in retinal fundus image. With regard to location methods, [5] located the center of the OD using the vasculature origin. They determined where all the vessels converged by means of a voting-type algorithm called fuzzy convergence. Another method that uses the convergence of the vessels to detect the OD center was proposed in [12]. The four main vessels originating from the OD were geometrically modeled using two parabolas, and the OD position was located as their common vertex. Inspired by previous works, Youssif et al. [6]. The OD center location was identified using the variance of intensity produced by the blood vessels within the OD. Hoover and Goldbaum [5]. Finding the OD can be used to decrease false positives in the detection of regions of retinal exudates [7]. These injuries are a diagnostic key to grading the risk of macular edema. An OD location method using three-independent location methods and a voting procedure is presented in [13]. Finally, a method for locating the OD using template matching techniques is presented in [14]. While in [14] used the Hough transform to detect the circular shape of the OD, in a complex optimization procedure is used.

\section{METHODOLOGIES}

Voting procedure is useful for selection of appropriate ODP. .Maximum varience methods used to calculates the statistical variance of every pixel. Low pass filter method is used for image smoothing and Maximum difference method is used to select OD pixel

\section{Voting Procedure:}

The final ODP is selected by taking into account the three previous candidate pixels and their location with respect to their average point (centroid). For this, a voting procedure comprising the following cases is applied.

$\square$ If the three OD candidate pixels are close to the centroid (closer than one-fifth of the image, maximum OD diameter estimation [11] The selected ODP is the centroid.

$\square$ If only two candidates are close to the centroid: the selected ODP is the average point in these two referred pixels.[1]

$\square$ Otherwise, the selected ODP is the candidate pixel obtained with the most reliable method $\square$ In this way the final ODP is locate using the voting procedure

\section{Maximum Variance Method:}

This method is based on the same properties as the previous one. It calculates the statistical variance for every pixel by using a $71 * 71$ centered window. On the other hand, a set of

"bright" pixels is obtained by automatic blue-channel thresholding according to the Otsu method [26].

Steps for finding ODP in maximum difference method are as follows:

a) Resize image.

b) Filtered by median filter $21 * 21$.

c) Thresholding.

d) Find standard deviation of image by $21 * 21$ window.

e) Finding co- ordinates of max value

f) Taking 10 bright Pixel.

g) Locate ODP.

The OD pixel returned by this method is the maximum variance pixel showing at least 10 "bright" pixels in its neighborhood

\section{Low-Pass Filter Method:}

The OD pixel of this method is the maximum gray-level pixel in a low-pass filtered image. Although the OD is usually the brightest area in a retinography, the pixel with the highest gray level could not be located within it. In many cases, this pixel may be inside other small bright regions. In order to smooth out these distractors, the image I is transformed to the frequency domain and filtered by the Gaussian low-pass filter defined[1]

In this method we are using the Gaussian low pass filter for smoothing and filtering with cut of frequency $25 \mathrm{~Hz}$.

$$
H(u, v)=\exp \left(\frac{-D^{2}(u, v)}{2 D_{0}^{2}}\right)
$$

Where, D $(\mathrm{u}, \mathrm{v})$ is the Euclidean distance between the points $(u, v)$ is origin of the frequency plane,

$\mathrm{S}$ and Do is the cutoff frequency with a value of $25 \mathrm{~Hz}$.

The highest gray-level pixel in the filtered image returned to the spatial domain is the result of this method.

\section{Maximum Difference Method:}

The OD usually appears as a bright region in eye fundus images. Moreover, the vascular tree formed by the"dark" blood vessels emerges in the disc.in OD the variation in maximum gray level occurs . This maximum is used by this method to select its OD pixel. A median filter

The OD pixel from this method is decided according to the following equation: Where Im denotes the filtered image.

$$
P_{\mathrm{MDM}}=\underset{(i, j)}{\arg }\left(\max \left\{\left(I_{M}\right)_{W}^{\mathrm{MAx}}(i, j)-\left(I_{M}\right)_{W}^{\mathrm{MIN}}(i, j)\right\}\right)
$$


Vol. 4, Special Issue 2, January 2017

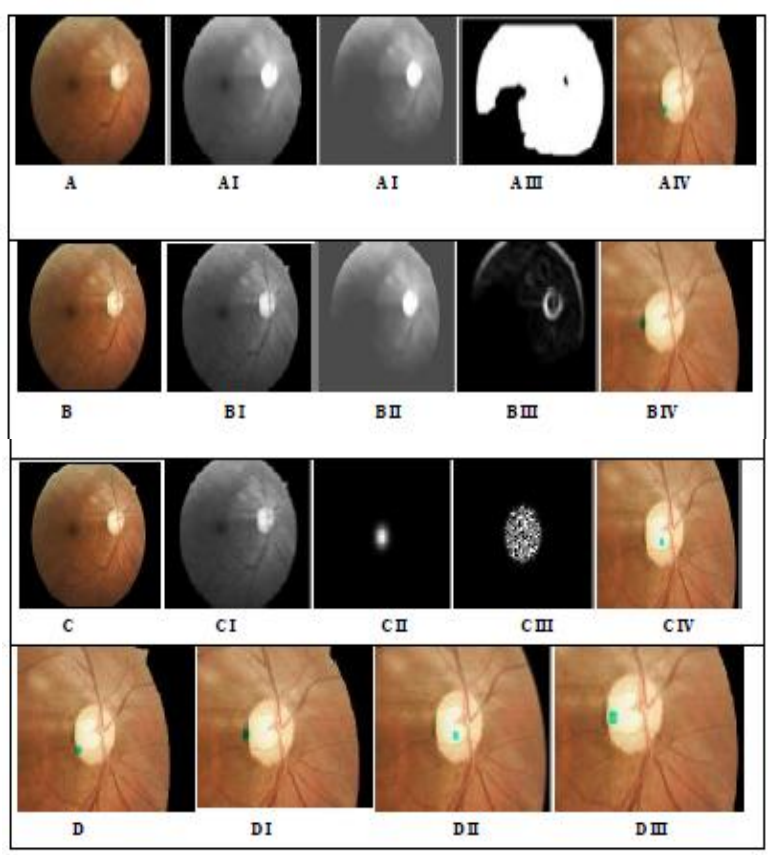

Fig.2: Experimental Result

The $I m_{W}^{M A x} \& I m_{W}^{M I N}$ are the maximum and the minimum values of the pixels in within a window of size $21 * 21$ centered on a pixel $(i, j) \ldots[1]$

1) Steps for finding ODP in maximum difference method.

a) Resize image.

b) Filtered by median filter $21 * 21$.

c) Thresholding.

d) Find $c(i, j)$ max and $c(i, j) m i n$ matrix.

e) Get difference image.

f) Locate ODP

\section{TESTING AND RESULTS}

The result of maximum difference method is $30 \%$.The result given by maximum variance method is $97 \%$. The result percentage observed in low pas filter method is $80 \%$. In this way maximum accuracy can be obtained by using the three methods to find the ODP.

\section{DISCUSSION AND CONCLUSION}

In this paper we are presenting the technique to find optic disc by using three different methods. Each method gives its own candidate pixel. By using this three ODP we locate exact optic disc pixel by using voting algorithm.

The result of the final ODP selection process is illustrated by the three examples of application of the methodology shown in Fig. In the first example, if in maximum difference method result shown outside the OD then the result will be discarded and the ODP is successfully selected. If pixels returned by the three methods are close, so the location of the ODP is the location of their centroid. If the three partial results are far from their centroid, so the final ODP is the OD pixel found by the maximum variance method.

\section{REFERENCES}

[1] Arturo Aquino, Manuel Emilio Gegúndez-Arias, and Diego Marín, "Detecting the Optic Disc Boundary in Digital Fundus Images Using Morphological, Edge Detection, and Feature Extraction Techniques"

[2] N. Patton, T. M. Aslam, T. MacGillivray, I. J. Deary, B. Dhillon, R. H. Eikelboom, K. Yogesan, and I. J. Constable, "Retinal image analysis: Concepts, applications and potential," Prog. Retin. Eye Res., vol. 25 , pp. 99-127, 2006.

[3] A. Singalavanija, J. Supokavej, P. Bamroongsuk, C. Sinthanayothin, S. Phoojaruenchanachai, and V. Kongbunkiat, "Feasibility study on computer-aided screening for diabetic retinopathy,” Jpn. J. Ophthalmol., vol. 50, pp. 361-366, 2006.

[4] A. W. Reza, C. Eswaran, and S. Hati, "Automatic tracing of optic disc and exudates from color fundus images using fixed and variable thresholds," J. Med. Syst., vol. 33, pp. 73-80, 2008.

[5] A. Hoover and M. Goldbaum, "Fuzzy convergence," in Proc. IEEE Comput.Soc. Conf. Comput.Vis.Pattern Recognit.,Santa Barbara, CA, 1998, pp. 716-721.

[6] A. Osareh, M. Mirmehdi, B. Thomas, and R. Markham, "Automated identification of diabetic retinal exudates in digital colour images," Br. J. Ophthalmol., vol. 87, pp. 1220-1223, 2003.

[7] T. Walter and J. C. Klein, "Automatic analysis of color fundus photographs and its application to the diagnosis of diabetic retinopathy," in Handbook of Biomedical Image Analysis. New York: Kluwer, 2005 , vol. 2, pp.315

[8] H. Li and O. Chutatape, "Automatic location of optic disc in retinal images,” in Proc. IEEE Int. Conf. Image Process., 2001, pp. 837840.

[10] Foracchia, M., Grisan, E., Ruggeri, A., "Detection of the optic disc in retinal images by means of a geometrical model of vessel structure", IEEE Trans. Med. Imag., vol. 23, no. 10, pp. 1189-1195, 2004.

[11] Yu, H., Barriga, E.S., Agurto, C., Echegaray, S., Pattichis, M.S., Bauman, W., Soliz, P. "Fast Localization and Segmentation of Optic Disk in Retinal Images Using Directional Matched Filtering and Level Sets", IEEE Trans. Inf. Tech. Biomed., vol. 16, no. 4, pp. 644-657, 2012.

[12] Marin, D., Aquino, A., Gegundez-Arias, M.E., Bravo, J.M. “A New Supervised Method for Blood Vessel Segmentation in Retinal Images by Using Gray-Level and Moment Invariants-Based Features", IEEE Trans. Med. Imag., vol. 30, no. 1, pp. 146-158, 2011.

[13] Fleming, A.D., Goatman, K.A., Philip, S., Olson, J.A., Sharp, P.F. "Automatic detection of retinal anatomy to assist diabetic retinopathy screening”, Phys. Med. Biol., vol. 52, pp. 331-345, 2007.

[14] Niemei jer, M., Abramoff, M.D., van Ginneken, B. "Fast detection of the optic disc and fovea in color fundus photographs", Med. Imag. Anal., vol. 13, pp. 859-870, 2009. 